\title{
Screened hydrodynamic interactions in ternary polymer solutions
}

\author{
Redouane Borsali $\left({ }^{1}, *\right)$, Thomas A. Vilgis $\left({ }^{2}\right)$ and Mustapha Benmouna (1,**) \\ (1) CERMAV-CNRS and University Joseph Fourier, P.O. Box 53x, F-38041 Grenoble Cedex, \\ France \\ (2) Max-Planck-Institut für Polymerforschung, P.O. Box 3148, D-6500 Mainz, Germany. \\ (Received 18 November 1992, revised 26 January 1993, accepted 11 February 1993)
}

\begin{abstract}
This paper discusses the effects of screened hydrodynamic interactions in ternary polymer solutions. The inclusion of a screening length $\xi_{H}$ in the Oseen tensor allows a good description of the wavevector dependence of the relaxation frequencies characterizing the mixture. In the limit of infinite screening length $\xi_{\mathrm{H}} \rightarrow \infty$, the full hydrodynamic interaction is recovered whereas for infinitely short length $\xi_{\mathrm{H}} \rightarrow 0$, the Rouse behavior is obtained. The method can be applied to arbitrary mixtures but for simplicity it is presented here in the symmetrical case.
\end{abstract}

\section{Introduction.}

Among the questions which are currently receiving a particular attention in the investigation of the dynamics of polymer mixtures is the effect of hydrodynamic interactions. Binary polymer/solvent systems have been already examined quite extensively by various authors [15] but the case of multicomponent mixtures and in particular those made of two polymers and a solvent has received only a limited attention from the point of view of the effect of hydrodynamic interaction usually modeled in the Oseen approximation, i.e.

$$
\mathbf{T}(\mathbf{R})=\left(1 / 8 \pi \eta_{0} R\right)\left(\mathbf{I}+\mathbf{R}: \mathbf{R} / R^{2}\right),
$$

where $\eta_{0}$ is the solvent viscosity, $\mathbf{R}$ is a vector distance between two monomers and the symbol : designates a tensor product. The purpose of this paper is to examine this question using a new method which includes the effect of screening in the hydrodynamic interaction through a dynamic correlation length $\xi_{\mathrm{H}}$ (c) which is function of the polymer concentration $c$.

This paper tries to resolve another controversy which has not yet been noticed in the literature, so far. Very often for studying the collective dynamics of semi-dilute and concentrated polymer solutions the mean-field expressions for the static structure factors have

(*) To whom correspondence should be addressed.

(**) Permanent Address: University of Tlemcen, Physics Department P.O. Box 119 Tlemcen, Algeria. 
been used, whereas in the same time the full long range hydrodynamic has been employed. This is, however, contradictory, since the mean field structure factors, calculated by the RPA become valid for large concentrations, but at the same time the long range interaction becomes screened from $1 / R$ to $(1 / R) \exp \left[-R / \xi_{\mathrm{H}}(c)\right]$. Therefore one claims that a more self-consistent model should be used, i.e. the use of the RPA (mean-field) structure factors and simultaneously a concentration dependent screened Oseen tensor :

$$
\mathbf{T}(\mathbf{R})=\frac{1}{8 \pi \eta_{0} R} \frac{\mathbf{I}+\mathbf{R}: \mathbf{R}}{R^{2}} \mathrm{e}^{-R / \xi_{\mathrm{H}}(c)}
$$

It is known from theoretical [6-11] and experimental [12-19] investigations that the dynamics of ternary polymer solutions can be characterized by two modes. The first one describes the relaxation of concentration fluctuations, and the second one is attributed to the relaxation of composition fluctuations. One can approximately write that the dynamic correlation function $S(q, t)$ is a sum of two exponentials :

$$
S(q, t)=A_{1}(q) \mathrm{e}^{-\Gamma_{1}(q) t}+A_{2}(q) \mathrm{e}^{-\Gamma_{2}(q) t}
$$

where the $A(q)$ 's and $\Gamma(q)$ 's are the amplitudes and the frequencies of the normal modes, respectively. The expressions of these quantities have been already given explicitly in general cases [6-11]. The amplitudes depend on the optical properties of the system whereas the frequencies are the same regardless of these properties. We shall examine only the latter ones focussing our attention on the effects of hydrodynamic interactions.

The physical meaning attributed to these two processes has been extensively discussed in various places [6-11]. We simply recall here that for symmetrical systems (the same molecular weight and the same composition of the two components), these processes are identified as the cooperative and the interdiffusive modes. In previous analyses of the quasi-elastic scattering data the Rouse model was used. The variations of the amplitudes and frequencies of these two processes [12-19] as a function of polymer concentration $c$, composition $x$, molecular weight $M_{w}$, etc.. were found qualitatively in good agreement with the theoretical predictions [6-11]. However, in certain cases and in particular for dilute solutions, some deviations were observed and have been attributed to the effects of hydrodynamic interaction.

To illustrate these aspects on a simple example, we consider a system at the volume fraction $\Phi$, containing two homopolymers which have the same degree of polymerization $N$, the same form factor $P(q)$ [or radius of gyration $R_{\mathrm{g}}$ ] embedded in a solvent presenting the same quality for both polymers : this means that the excluded volume parameter $v$ of polymer/solvent is also the same for both polymers. The chemical difference between them is characterized by a nonzero Flory [20] $\chi$-parameter. To simplify the problem further, we choose the symmetrical case where the composition $x$ is equal to $1 / 2$. Here, we refer to the concentration either by $c$ expressed in $\mathrm{g} / \mathrm{cm}^{3}$, or by $\Phi$ the volume fraction assuming that both monomer species in the mixture have the same density.

Under such assumptions, the relevant quantities that are of interest and which can be measured experimentally by the use of quasi elastic light scattering and/or neutron spin echo technique, are :

The cooperative relaxation frequency :

$$
\Gamma_{1}(q)=\Gamma_{\mathrm{C}}(q)=q^{2} k_{\mathrm{B}} T \frac{M(q)+M^{\prime}(q)}{S(q)+S^{\prime}(q)}
$$

and the interdiffusive one :

$$
\Gamma_{2}(q)=\Gamma_{\mathrm{I}}(q)=q^{2} k_{\mathrm{B}} T \frac{M(q)-M^{\prime}(q)}{S(q)-S^{\prime}(q)}
$$


where $q$ is the amplitude of the scattering wavevector, and $k_{\mathrm{B}} \mathrm{T}$ the product of the Boltzmann constant with the absolute temperature. The derivation of these results has been reproduced elsewhere (see for example Ref. [21]) and therefore there is no need to give more details here. The partial structure factors $S(q)$ and $S^{\prime}(q)$ were also calculated for the present system and the results are [21]:

$$
\begin{aligned}
S(q) & =\frac{(\Phi N P(q) / 2)[1+v \Phi N P(q) / 2]}{[1+(v+\chi / 2) \Phi N P(q)][1-\chi \Phi N P(q) / 2]} \\
S^{\prime}(q) & =-\frac{(v+\chi) \Phi^{2} N^{2} P^{2}(q) / 4}{[1+(v+\chi / 2) \Phi N P(q)][1-\chi \Phi N P(q) / 2]}
\end{aligned}
$$

The mobility matrix is symmetric and $M(q), M^{\prime}(q)$ represent its elements and they can be written including both the Rouse and the hydrodynamic terms as follows:

$$
\begin{aligned}
M(q) & =\frac{\Phi}{2 \zeta}+\frac{1}{(2 \pi)^{2} \eta_{0}} \int_{0}^{\infty} \mathrm{d} k F\left(\frac{k}{q}\right) S(k) \\
M^{\prime}(q) & =\frac{1}{(2 \pi)^{2} \eta_{0}} \int_{0}^{\infty} \mathrm{d} k F\left(\frac{k}{q}\right) S^{\prime}(k) .
\end{aligned}
$$

$\zeta$ is the friction coefficient per monomer, and it is related to the solvent viscosity through the draining parameter $\left(\zeta / \eta_{0} a\right)=3 \pi, a$ being the length of the unit segment. The function $F(x)$ is obtained from the angular integration of the screened Oseen tensor [1-5] :

$$
F(x)=x^{2}\left\{\frac{x^{2}+1+1 /\left(q^{2} \xi_{\mathrm{H}}^{2}\right)}{4 x} \log \frac{(1+x)^{2}+1 /\left(q^{2} \xi_{\mathrm{H}}^{2}\right)}{(1-x)^{2}+1 /\left(q^{2} \xi_{\mathrm{H}}^{2}\right)}-1\right\} ; \quad x=k / q .
$$

It is easily seen that the function $F(x)$ contains the two extreme limits. Firstly if $\xi_{\mathrm{H}} \rightarrow \infty$; i.e. the hydrodynamic interaction is fully developed, the Kawasaki form is recovered [3]. On the other hand, if $\xi_{H}=0$, the hydrodynamic effects are screened out down to the length scale of the monomer segment, $F(x)=0$ and we are left with the Rouse mobility $M(q)=\Phi / 2 \zeta$ see equation (7). This model is formally different from the screened hydrodynamic model of Roby and Joanny [11]. The latter is based on the idea of Richter et al. (5) to replace the viscosity $\eta_{0}$ by a renormalized $q$-dependent viscosity $\eta(q)$ with the additional assumption that the viscosity is infinite on the large scale. Nevertheless the present model can also be regarded as a generalization to a space dependent viscosity as a way to express the hydrodynamic screening with the introduction of a hydrodynamic characteristic length $\xi_{\mathrm{H}}$. In this representation, $\eta_{0}$ is replaced by $\eta(q)=\eta_{0}\left[1+\left(q \xi_{\mathrm{H}}\right)^{-2}\right]$ which tends to $\eta_{0}$ at high values of $q \xi_{\mathrm{H}}$ and to infinity when $q \xi_{\mathrm{H}}$ goes to zero, consistently with the model of Roby and Joanny. Since the variation of $\xi_{\mathrm{H}}$ with the polymer concentration is not precisely known, we shall use it as a parameter in the examples to be discussed below.

Substituting equations (7) and (8) into equations (3) and (4) leads to

$$
\Gamma_{\mathrm{C}}(q)=q^{2} \frac{k_{\mathrm{B}} T}{S(q)+S^{\prime}(q)}\left\{\frac{\Phi}{2 \zeta}+\frac{1}{(2 \pi)^{2} \eta_{0}} \int_{0}^{\infty} \mathrm{d} k F\left(\frac{k}{q}\right)\left[S(k)+S^{\prime}(k)\right]\right\}
$$

and

$$
\Gamma_{\mathrm{I}}(q)=q^{2} \frac{k_{\mathrm{B}} T}{S(q)-S^{\prime}(q)}\left\{\frac{\Phi}{2 \zeta}+\frac{1}{(2 \pi)^{2} \eta_{0}} \int_{0}^{\infty} \mathrm{d} k F\left(\frac{k}{q}\right)\left[S(k)-S^{\prime}(k)\right]\right\}
$$




\section{Discussions and conclusions.}

To illustrate the effect of screening of the hydrodynamic interaction, we have considered the frequency $\Gamma_{\mathrm{I}}(q)$ and plotted in figure 1 the variation of $\Gamma_{\mathrm{I}}(q) /\left[q^{2}\left(k_{\mathrm{B}} T / N \zeta\right)\right]$ as a function of $q$ for several values of the screening length $\xi_{\mathrm{H}}$. The curve a is plotted in the limit of $\xi_{\mathrm{H}}=\infty$, which means that the hydrodynamic interaction is fully developed through the unscreened Oseen tensor. The curves $b$ and c correspond to $\xi_{\mathrm{H}}=60 \AA$ and $5 \AA$ respectively. They show that the strength of hydrodynamic screening increases significantly when $\xi_{\mathrm{H}}$ decreases, especially in the lower $q$ range. The curve $d$ represents the Rouse behavior which is obtained in the limit of $\xi_{\mathrm{H}}=0$ and describes the dynamics in the absence of long range hydrodynamic backflow effects. These plots were made by choosing the volume fraction of the polymers $\Phi$ such as $v \Phi N=10$ which is about 10 times the overlap concentration $\Phi *$ corresponding roughly to $v \Phi * N=1$. There are shown for the sake of illustration of the effect of screening when $\xi_{\mathrm{H}}$ decreases from infinity (curve a, non screening) to zero (curve d,

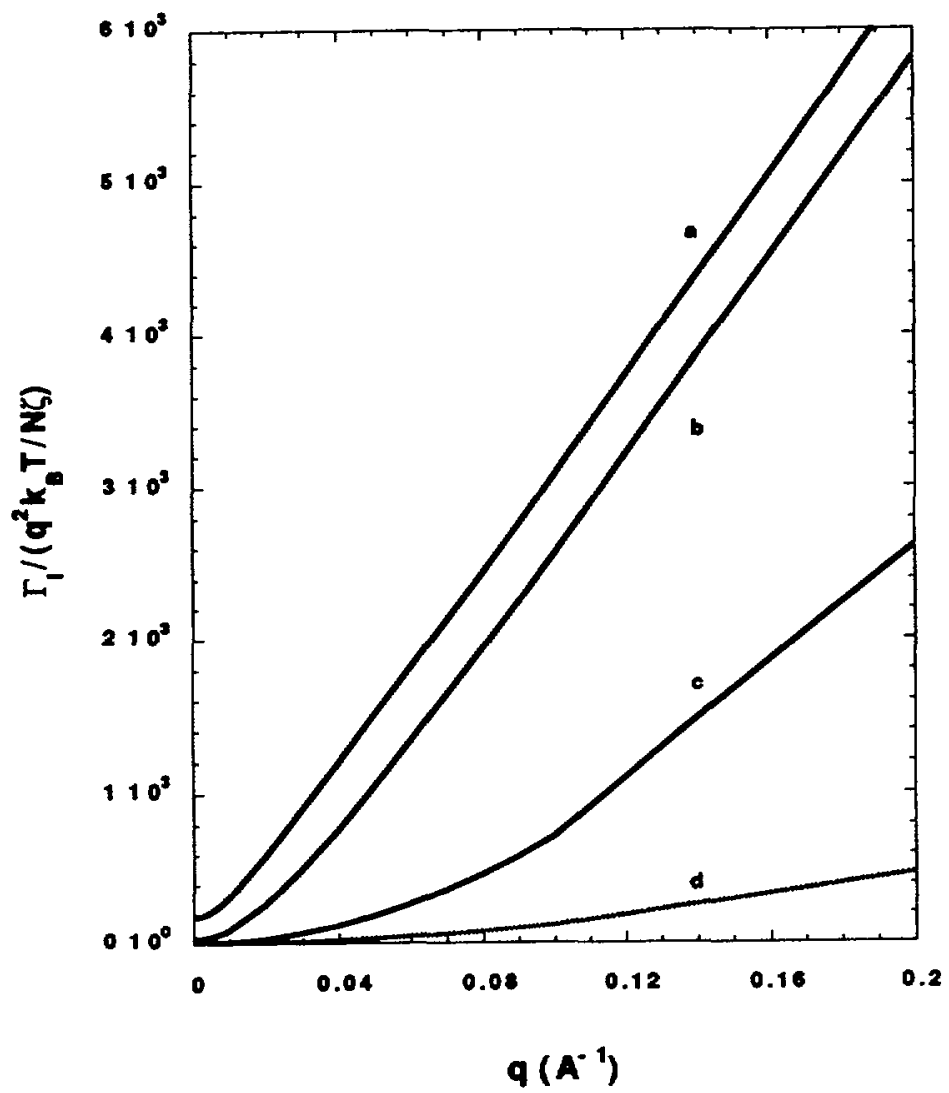

Fig. 1. - Variation of the normalized relaxation frequency for the interdiffusive mode $\Gamma_{\mathrm{I}}(q) /\left[q^{2}\left(k_{\mathrm{B}} T / N \zeta\right)\right]$ as a function of $q$ for several values of the screening length $\xi_{\mathrm{H}}$. a) $\xi_{\mathrm{H}}=\infty$ (no screening); b) $\xi_{\mathrm{H}}=60 \AA$; c) $\xi_{\mathrm{H}}=5 \AA$ and d) $\xi_{\mathrm{H}}=0$ (complete screening). The concentration corresponds to $v \Phi N=10$; the draining parameter $\left(\zeta / \eta_{0} a\right)=3 \pi ;(a$ being the monomer diameter); the Flory interaction parameter is $\chi^{\prime v}=0.1$. 
complete screening). For the curves $b$ and $c$ the values of $\xi_{\mathrm{H}}$ were chosen arbitrarily due to the lack of an exact expression which gives the values of $\xi_{\mathrm{H}}$ as function of the concentration. Similar observations [22] were made elsewhere in the investigation of the single labeled chains dynamics in a matrix of identical but unlabeled chains and a solvent. In this work, it was found that, in the intermediate $q$ range, the relaxation rate of the concentration fluctuations $\Gamma^{-1}(q)$ satisfies the limit $\Gamma(q) /\left[q^{3}\left(k_{\mathrm{B}} T / \eta_{0}\right)\right]=\alpha=$ Constant when $q \xi_{\mathrm{H}} \gg 1$. The constant $\alpha$ depends on the quality of the solvent and on the hydrodynamic model. In good solvent conditions, $\alpha$ is found to be equal to 0.071 in the preaveraged Oseen tensor, and to 0.079 in its non-preaveraged version. In the theta solvent condition, $\alpha$ is obtained as $1 / 6 \pi=0.053 \mathrm{using}$ preaveraged Oseen tensor and as $1 / 16=0.062$ in its non-preaveraged version. To see whether a similar observation can be made here, we have plotted in figure 2 the variation of $\Gamma_{\mathrm{I}}(q) /\left[q^{3}\left(k_{\mathrm{B}} T / \eta_{0}\right)\right]$ as a function of $q$ in the upper $q$ range and in the same conditions as in figure 1. One observes that the plateau is reached much faster in the absence of screening. Indeed as $\xi_{\mathrm{H}}$ decreases, the screening becomes more effective and the rise of the curves $\Gamma_{\mathrm{I}}(q) /\left[q^{3}\left(k_{\mathrm{B}} T / \eta_{0}\right)\right]$ versus $q$ is slower. Although the limiting value of the constant $\alpha$ is not completely reached in the presence of full hydrodynamic interaction (curve a), one observes that the constant $\alpha$ tends to 0.07 which is closer to the result in good solvent condition and preaveraged Oseen tensor. This is somewhat surprising in view of the approximations used and in particular the full Oseen tensor with various hydrodynamic screening lengths, the

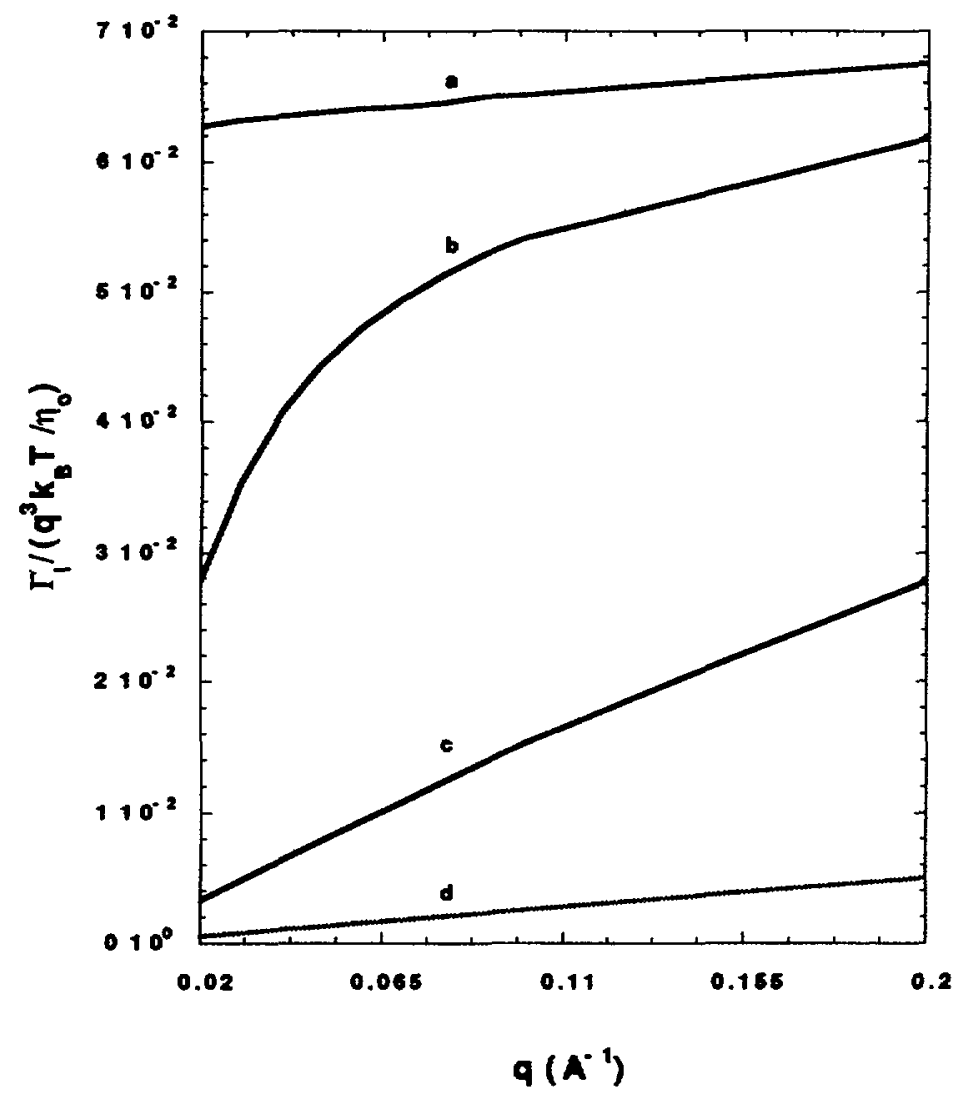

Fig. 2. - Variation of the normalized relaxation frequency for the interdiffusive mode $\Gamma_{\mathrm{I}}(q) /\left[q^{3}\left(k_{\mathrm{B}} T / \eta_{0}\right)\right]$ as a function of $q$ in the upper $q$-range in the same condition as figure 1 . 
random phase approximation in the calculation of the structure factors and the Debye function in the form factors. It should be recalled however that as the concentration of the polymer increases, not only hydrodynamic interaction is screened but the excluded volume effects as well, although the screening lengths for both interactions are not necessarily the same in the front factor but in their concentration scaling dependence [23].

In conclusion this paper addressed the effects of hydrodynamic screening on the relaxation frequencies of the concentration and the composition fluctuations in ternary polymer solutions. It is observed that as the range of hydrodynamic screening decreases, the variation of the relaxation frequencies as a function of $q$ shows a transition from a Zimm-like behavior to a Rouse-like behavior. In the intermediate $q$ range the quantity $\alpha(q)=\Gamma(q) /\left[q^{3}\left(k_{\mathrm{B}} T / \eta_{0}\right)\right]$ depends substantially on the strength of hydrodynamic screening. When $\xi_{\mathrm{H}}$ is infinitely large, the screening is very weak and $\alpha(q)$ tends to reach quickly its plateau limit which is around 0.07 . As $\xi_{\mathrm{H}}$ decreases, $\alpha(q)$ increases slowly indicating a strong influence of the Rouse term. The limit of the constant $\alpha=0.07$ seems to favor the model of preaveraged Oseen tensor in good solvent conditions.

\section{Acknowledgments.}

R. B. would like to acknowledge the financial support from the Sonderforschungbereich (SFB262) and the hospitality of the Max-Planck-Institut für Polymerforschung, Mainz where this work was performed. M. B. thanks the Max-Planck-Gessellschaft.

\section{References}

[1] YAMAKAwA A., Modern theory of polymer solutions (Harper \& Row, New York, 1971).

[2] De Gennes P. G., Scaling Concepts in Polymer Physics (Cornell University Press, Ithaca, 1979).

[3] DoI M., Edwards S. F., Dynamic Theory of Polymer Solutions (Oxford University Press, 1986).

[4] DES CloizeauX J., Jannink G., Les polymères en Solution : leur modélisation et leur structure (Les Editions de Physique, 1987).

[5] Richter D., Ewen B., Binder K., J. Phys. Chem. 88 (1984) 6618.

[6] AkCasu Z. A., Dynamic Light Scattering, W. Brown Ed. (Oxford University Press, 1992).

[7] AkCasu Z. A., NÄGEle G., KleIN R., Macromolecules 24 (1991) 4408.

[8] Benmouna M., Benoit H., Duval M., AKCasu A. Z., Macromolecules 20 (1987) 1107.

[9] Borsali R., VIlgis T. A., J. Chem. Phys. 93 (1990) 3610.

[10] Vilgis T. A., Benmouna M., J. Chem. Phys. 15 (1991) 1276.

[11] ROBY F., JOANnY J. F., Macromolecules 25 (1992) 4612.

[12] Borsali R., Duval M., Benmouna M., Polymer 30 (1989) 610.

[13] Borsali R., Duval M., Benmouna M., Macromolecules 30 (1989) 610.

[14] Giebel L., Borsali R., Fischer E. W., Meier G., Macromolecules 24 (1991) 4054.

[15] Giebel L., Borsali R., Fischer E. W., Benmouna M., Macromolecules 25 (1992) 4378.

[16] BROWN W., ZHOU P., Macromolecules 22 (1989) 4031.

[17] Nemoto N., Inoue T., Makita Y., Tshunashima Y., Kurata M., Macromolecules 18 (1985) 2516.

[18] Daivis P. J., Pinder D. N., Callaghan P. T., Macromolecules 25 (1992) 170.

[19] Wheeler L. M., Lodge T. P., Hanley B., Tirell M., Macromolecules 20 (1987) 1120.

[20] FLORY P., Introduction to Polymer Chemistry (Cornell University Press, Ithaca, 1965).

[21] Benmouna M., Benmansour Z., Benoit H., Fischer E. W., Vilgis T. A., J. Polym. Sci. Polym. Phys. Ed. 30 (1992) 733.

[22] Akcasu A. Z., Benmouma M., Han C. C., Polymer 21 (1980) 866.

[23] DE GENNES P. G., Macromolecules 9 (1976) 594. 